\title{
Numerical Investigation of Supersonic Nozzle Flow Separation
}

\author{
Q. Xiao* and H. M. Tsai \\ National University of Singapore, Singapore 119260, Republic of Singapore \\ and \\ D. Papamoschou辛 \\ University of California, Irvine, Irvine, California 92697-3975 \\ DOI: $\underline{10.2514 / 1.20073}$
}

\begin{abstract}
Separation of supersonic flow in a planar convergent-divergent nozzle with moderate expansion ratio is investigated by solving the Reynolds-averaged Navier-Stokes equations with a two-equation $k$ - $\omega$ turbulence model. The focus of the study is on the structure of the fluid and wave phenomena associated with the flow separation. Computations are conducted for an exit-to-throat area ratio of 1.5 and for a range of nozzle pressure ratios. The results are compared with available experimental data in a nozzle of the same geometry. The flow separates by the action of a lambda shock, followed by a succession of expansion and compression waves. For $1.5<\mathrm{NPR}<2.4$, the computation reveals the possibility of asymmetric flow structure. The computationally obtained asymmetric flow structures are consistent with previous experimental flow visualizations studies. In addition, other flow features such as shock location and wall pressure distributions are also in good agreement with the experimental data. The present study provides new information that confirms earlier conjectures on the flow-wave structure relevant to the instability of the separated flow in convergent-divergent nozzles of moderate expansion ratio.
\end{abstract}

\section{Nomenclature}

$A$

$e$

$H$

$h$

$M$

NPR

$P$

$P_{0}$

$R$

$T$

$u, v, w=$

$x \quad=$

$y$

$\gamma=$

$\theta \quad=$

$\mu \quad=$

$\varphi$

Subscripts

$0=$ total

$1=$ immediately before the Mach stem

$2=$ immediately after the Mach stem

$a \quad=$ ambient

$c \quad=$ centerline

$e \quad=$ nozzle exit

$s \quad=$ shock location

$t=$ throat

Presented as Paper 4640 at the 35 th AIAA Fluid Dynamics Conference and Exhibit, Toronto, Canada; received 14 September 2005; revision received 20 April 2006; accepted for publication 29 June 2006. Copyright (C) 2006 by the authors. Published by the American Institute of Aeronautics and Astronautics, Inc., with permission. Copies of this paper may be made for personal orinternal use, on condition that the copier pay the $\$ 10.00$ per-copy fee to the Copyright Clearance Center, Inc., 222 Rosewood Drive, Danvers, MA 01923;include the code0001-1452/07 \$10.00in correspondence with the CCC.

* Research Scientist, Temasek Laboratories.

${ }^{\dagger}$ Principal Research Scientist, Temasek Laboratories. Member AIAA.

${ }^{\ddagger}$ Professor, Department of Mechanical and Aerospace Engineering. Associate Fellow AIAA.

\section{Introduction}

EPARATION of supersonic flow in a convergent-divergent nozzle is a fundamental fluid phenomenon that affects a large variety of applications, from fuel systems to aircraft engine nozzles. When a supersonic nozzle is operated at pressure ratios well below its design point, a shock forms inside the nozzle and flow downstream of the shock separates from the nozzle walls. Even though flow separation is typically viewed as an undesirable occurrence, it may have some interesting applications in the area of fluid mixing. Specifically, past work at the University of California, Irvine has shown that flow exiting a severely overexpanded nozzle exhibits a strong instability that enhances mixing of the flow itself and can also be used as a fluidic actuator to enhance mixing of an adjacent flow [13].

A large portion of the literature on nozzle flow separation focuses on prediction of the separation location in rocket nozzles. A review paper by Morrisette and Goldberg [4], based on a variety of experimental results, concludes that computational methods, like those proposed by Reshotoko and Tucker [5] , give reasonable predictions of turbulent flow separation in a nozzle with a large divergence angle. However, generic methods for boundary-layer separation prediction cannot capture entire series of events inside a nozzle. Recently, a theoretical model proposed by Romine [6] helps fill this gap. For shocks with moderate Mach numbers (less than $2.25)$, Romine postulates that the jet flow emerging from the shock is above ambient pressure and adjusts to the ambient pressure via a gradual underexpansion. It is important to note that this argument applies only in the vicinity of the centerline of the nozzle, the shock of which is normal or close to normal, and not in regions nearer to the walls. On the walls, there is general consensus that the flow adjusts to the ambient pressure via a gradual compression.

Previous numerical studies of separated nozzle flows, such as studies by Hunter [7], Carlson [8], and Xiao et al. [9,10] show that there is excellent agreement with available experimental data. In the study of Hunter, which is a combined computational and experimental investigation, two distinct separation regimes were found in a planar nozzle with an area ratio of exit to the throat area $A_{e} / A_{t}$ of 1.8 . For NPR $<1.8$, the flow shows three-dimensional separation with partial reattachment. Fully detached two-dimensional separation is found for NPR $>2.0$. The underexpansion of flow after the main shock, postulated by Romine [6], is evident from 
the computed Mach number contours, although this was not explicitly stated.

The present study focuses more on the flow structure than on prediction methods. It is motivated in part by the large instability associated with supersonic flow separation, which can be used as an excitation method for mixing enhancement [1-3]. In this sense, we attempt to reproduce and expand on the experimental results of Papamoschou and Zill [11], who experimentally investigated the supersonic nozzle flow separation inside such planar convergentdivergent nozzles that produce flows for such purposes. Their study shows that for area ratio $A_{e} / A_{t} \geq 1.4$ and nozzle pressure ratio NPR $>1.4$, the flow pattern is asymmetric, characterized by a lambda shock with one foot always larger than the other. This asymmetry does not flip during a given test run, but can change sides from one run to the next. The flow downstream of the Mach stem was observed from schlieren pictures to expand to near-sonic speed and exhibit an alternation of subsonic and supersonic regions. The experimental measurements of centerline pressure distribution are qualitatively consistent with this observation. It was also found that for large $A_{e} / A_{t}$ and NPR, the shock is unsteady but does not emit resonant acoustic tones.

A different type of asymmetric flow separation was previously investigated in the ONERA S8Ch wind tunnel by Bourgoing and Reijasse [12] and Reijasse and Bourgoing [13]. Steady and unsteady asymmetric flow separations in supersonic planar overexpanded nozzles were induced by a second variable throat located downstream of the test section. Depending on the setting of the second throat, stable symmetric and asymmetric lambda shock patterns were obtained in the test section. The asymmetries of the separation and associated shock structure could be conditioned by

\section{Numerical Method}

The Reynolds-averaged governing equations for unsteady compressible turbulent flow with a two-equation $k$ - $\omega$ turbulence model are expressed as follows:

$$
\frac{\partial}{\partial t} \int_{\Omega} \boldsymbol{W} \mathrm{d} \Omega+\oint_{\partial \Omega}\left(\boldsymbol{F}_{c}-\boldsymbol{F}_{d}\right) \mathrm{d} S=\int_{\Omega} S \mathrm{~d} \Omega
$$

The vector $\boldsymbol{W}$ contains the conservative variables:

$$
\boldsymbol{W}=\{\rho, \quad \rho u, \quad \rho v, \quad \rho w, \quad \rho E, \quad \rho k, \quad \rho \omega\}^{T}
$$

where $\rho$ is the density, $u, v$, and $w$ are the three Cartesian velocity components, and $e_{0}$ is the total energy of the flow, given by

$$
e_{0}=e+\frac{1}{2}\left(u^{2}+v^{2}+w^{2}\right)
$$

The flux vectors in Eq. (1) comprise the inviscid convective fluxes $\boldsymbol{F}_{c}$ and the diffusive fluxes $\overline{\boldsymbol{F}}_{d}$. For the convective fluxes, we include the pressure term

$$
\boldsymbol{F}_{c}=\left\{\begin{array}{ccc}
\rho u & \rho v & \rho w \\
\rho u u+p & \rho u v & \rho u w \\
\rho v u & \rho v v+p & \rho v w \\
\rho w u & \rho w v & \rho w w+p \\
\rho e_{0} u+p u & \rho e_{0} v+p v & \rho e_{0} w+p w \\
\rho k u & \rho k v & \rho k w \\
\rho \omega u & \rho \omega v & \rho \omega w
\end{array}\right\}
$$

For the diffusive fluxes, we have

$$
\boldsymbol{F}_{d}=\left\{\begin{array}{ccc}
0 & 0 & 0 \\
\tau_{x x} & \tau_{x y} & \tau_{x z} \\
\tau_{y x} & \tau_{y y} & \tau_{y z} \\
\tau_{z x} & \tau_{z y} & \tau_{z z} \\
u \tau_{x x}+v \tau_{x y}+w \tau_{x z}-q_{x} & u \tau_{y x}+v \tau_{y y}+w \tau_{y z}-q_{y} & u \tau_{z x}+v \tau_{z y}+w \tau_{z z}-q_{z} \\
\mu^{*} \frac{\partial k}{\partial x} & \mu^{*} \frac{\partial k}{\partial y} & \mu^{*} \frac{\partial k}{\partial z} \\
\mu^{*} \frac{\partial \omega}{\partial x} & \mu^{*} \frac{\partial \omega}{\partial y} & \mu^{* \frac{\partial \omega}{\partial z}}
\end{array}\right\}
$$

the state of the upstream boundary layers. Streamwise movements and evolution of the shock wave were observed by high-speed shadowgraphs. The measurement of the wall fluctuating pressure shows two low-frequency phenomena, with one large energetic frequency band around $60 \mathrm{~Hz}$ probably due to the fluctuations of the separated flow, and another less energetic peak at $200 \mathrm{~Hz}$ due to shock-induced oscillation. The numerical simulation of the same planar nozzle was examined by Deck et al. [14] using steady Reynolds-averaged Navier-Stokes (RANS) equations with the Spalart-Allmaras turbulence model. Depending on the different initial field used, two asymmetric and one symmetric shock structures were computed.

The aim of the present paper is to numerically study in detail the flow structure and wave structures in the vicinity of the separation shock, for the purpose of understanding the salient physics and eventually modeling the flow instability downstream of the shock. The experiment by Papamoschou and Zill [11] is used as a basis for comparison in which, unlike the study of Bourgoing and Reijasse [12], Reijasse and Bourgoing, [13], and Deck et al. [14], symmetric or asymmetric separated flow are naturally occurring, depending on the nozzle pressure ratio. In the following sections, the governing equations and the numerical methods are briefly outlined, followed by a discussion of the numerical results and concluding remarks. where

$$
\mu^{*}=\mu_{L}+\sigma^{*} \mu_{T}
$$

where $\mu_{L}$ is the laminar viscosity, $\mu_{T}$ is turbulent eddy viscosity, $\sigma^{*}$ is turbulent closure constant equal to 0.5 , and $\tau$ is the stress tensor. The source term $S$ is defined as

$$
\boldsymbol{S}=\left\{\begin{array}{c}
0 \\
0 \\
0 \\
0 \\
0 \\
\tau_{i j} \frac{\partial u_{i}}{\partial x_{j}}-\beta^{*} \rho \omega k \\
\frac{\alpha \omega}{k} \tau_{i j} \frac{\partial u_{i}}{\partial x_{j}}-\beta \rho \omega^{2}
\end{array}\right\}
$$

with closure constants of $\alpha=5 / 9, \beta=0.075$, and $\beta^{*}=0.09$. The shear stresses and heat fluxes follow the customary Newton and Fourier laws: 


$$
\begin{gathered}
\tau_{x x}=2 \mu^{*} \frac{\partial u}{\partial x}-\frac{2}{3} \mu^{*}\left(\frac{\partial u}{\partial x}+\frac{\partial v}{\partial y}+\frac{\partial w}{\partial z}\right)-\frac{2}{3} \rho k \\
\tau_{y y}=2 \mu^{*} \frac{\partial v}{\partial y}-\frac{2}{3} \mu^{*}\left(\frac{\partial u}{\partial x}+\frac{\partial v}{\partial y}+\frac{\partial w}{\partial z}\right)-\frac{2}{3} \rho k \\
\tau_{z z}=2 \mu^{*} \frac{\partial w}{\partial z}-\frac{2}{3} \mu^{*}\left(\frac{\partial u}{\partial x}+\frac{\partial v}{\partial y}+\frac{\partial w}{\partial z}\right)-\frac{2}{3} \rho k \\
\tau_{x y}=\tau_{y x}=\mu^{*}\left(\frac{\partial u}{\partial y}+\frac{\partial v}{\partial x}\right) \quad \tau_{x z}=\tau_{z x}=\mu^{*}\left(\frac{\partial w}{\partial x}+\frac{\partial u}{\partial z}\right) \\
\tau_{y z}=\tau_{z y}=\mu^{*}\left(\frac{\partial v}{\partial z}+\frac{\partial w}{\partial y}\right) \quad q_{x}=-\left(k_{L}+k_{T}\right) \frac{\partial T}{\partial x} \\
q_{y}=-\left(k_{L}+k_{T}\right) \frac{\partial T}{\partial y} \quad q_{z}=-\left(k_{L}+k_{T}\right) \frac{\partial T}{\partial z}
\end{gathered}
$$

where $k_{L}$ is the laminar thermal conductivity and $k_{T}$ is the turbulent thermal conductivity.

The total enthalpy $h_{0}$ is defined by

$$
h_{0}=h+\frac{1}{2}\left(u^{2}+v^{2}+w^{2}\right)
$$

Using the following relations for a perfect gas,

$$
e=c_{v} T, \quad h=c_{P} T, \quad R=c_{p}-c_{v}, \quad \gamma=c_{p} / c_{v}
$$

and the ideal gas law

$$
p / \rho=R T
$$

the pressure and total enthalpy can be evaluated as

$$
p=(\gamma-1) \rho\left[e_{0}-\frac{1}{2}\left(u^{2}+v^{2}+w^{2}\right)\right]
$$

and

$$
h_{0}=e_{0}+p / \rho
$$

where $c_{p}$ and $c_{v}$ are the specific heats at constant pressure and volume, respectively. The temperature is then given by

$$
T=\frac{\gamma}{c_{p}(\gamma-1)} \frac{p}{\rho}
$$

The coefficient of laminar viscosity $\mu_{L}$ is obtained by Sutherland's formula:

$$
\frac{\mu_{L}}{\mu_{\text {ref }}}=\left(\frac{T}{T_{\text {ref }}}\right)^{\frac{3}{2}} \frac{T_{\text {ref }}+110.3}{T+110.3}
$$

The basic numerical method used to solve the preceding system of equations follows that described in detail by Sadeghi et al. [15]. A cell-centered finite volume scheme is used to discretize the governing equations. The $k$ and $\omega$ variables are defined at the cell centers, in a similar fashion to the main flow quantities. A central difference scheme is used to discretize the diffusive terms. A scalar dissipation Jameson-Schmidt-Turkel scheme is used for the convective terms in the Navier-Stokes equation. A second-order MUSCL-type (monotone upstream-centered scheme for conservation laws) upwind scheme is applied for the $k-\omega$ equations. Parallel computation is achieved through the implementation of messagepassing interface protocol. The computational domain is decomposed by subdivisions of the structured grids. Each block is considered as a single entity, and only flow and turbulence quantities at the block boundaries need to be exchanged.

After discretization in space, the governing equations are reduced to a set of ordinary differential equations with only derivatives in time, which can be readily solved using an explicit multistage Runge-Kutta type of scheme. Here, a five-stage scheme is used. The artificial dissipation is updated at stages 1, 3, and 5. Local pseudo time-stepping is used to advance the flow solution at the local maximum speed. Residual smoothing is applied at stages 1, 3, and 5 to increase the stability limit. A multigrid method is adopted to
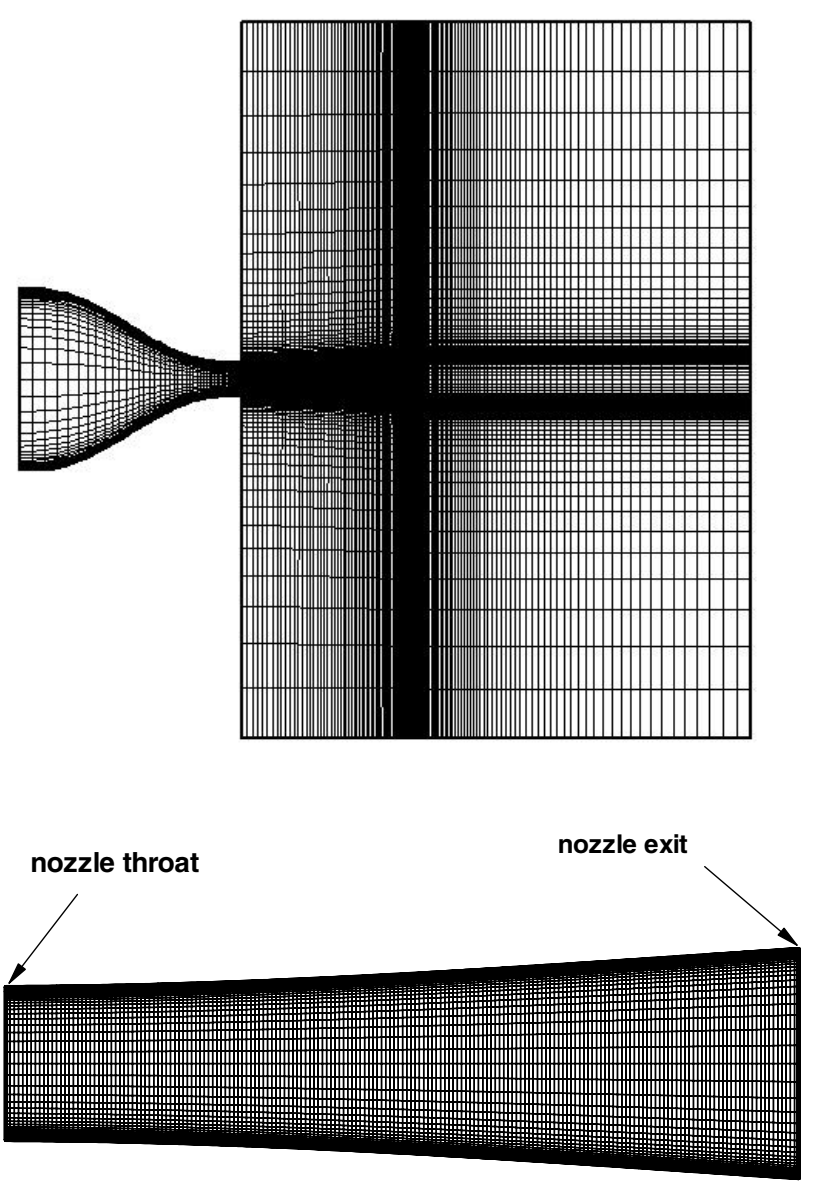

Fig. 1 Computational domain and grid distribution: a) overall computational domain, both inside and outside the nozzle and b) mesh distribution inside the nozzle.

accelerate the convergence of the solution, and a dual time-stepping method is used for time-accurate unsteady time marching.

The same case as the planar 2-D convergent-divergent supersonic nozzle tested by Papamoschou and Zill [11] is studied here. The nozzle area ratio $A_{e} / A_{t}$ is fixed at 1.5 and the nozzle divergence angle is $3.89 \mathrm{deg}$, the same value as in the experiment. Experiments were conducted for $1.27 \leq \mathrm{NPR} \leq 1.61$. Computations are made for NPRs between 1.27 and 2.4 and compared with the available experimental data.

The computational domain includes the domain inside the nozzle and an ambient region around the outer surface and downstream of the nozzle, with ten throat heights downstream, upstream, and normal to the jet axis. Figure 1 shows the geometry and grid distribution inside the nozzle. The grid density is higher in the divergent part of the nozzle to improve the resolution for capturing shocks. The grid is clustered along the top and bottom walls. Gridindependence testing was conducted for $119 \times 45,237 \times 85$, and $355 \times 127$ resolutions inside the nozzle, and the result (not shown here) shows that a $237 \times 85$ grid was adequately fine to capture the main flow characteristics inside the nozzle. The total number of grids used for the whole computational domain is 41,585 . For a Reynolds number based on the throat height of $5.5 \times 10^{6}$, the minimum first grid point from the wall gives a $y^{+}<1$.

Boundary conditions are imposed as follows: total pressure and total temperature at the nozzle inlet are set to be $p_{0}=\mathrm{NPR} \times p_{a}$ and $T_{t}=T_{a}$, respectively, where $p_{a}$ is the ambient pressure equal to $14.85 \mathrm{psi}$ and $T_{a}$ is the ambient temperature set at $290 \mathrm{~K}$. No-slip boundary conditions are specified for the nozzle top and bottom walls, and the ambient pressure and freestream Mach number $(M=0.1)$ are set along the outer surface of the computational domain. Pure air is simulated with $\gamma=1.4$.

The simulation was initially conducted with an unsteady timeaccurate dual time-stepping method. However, the convergence 


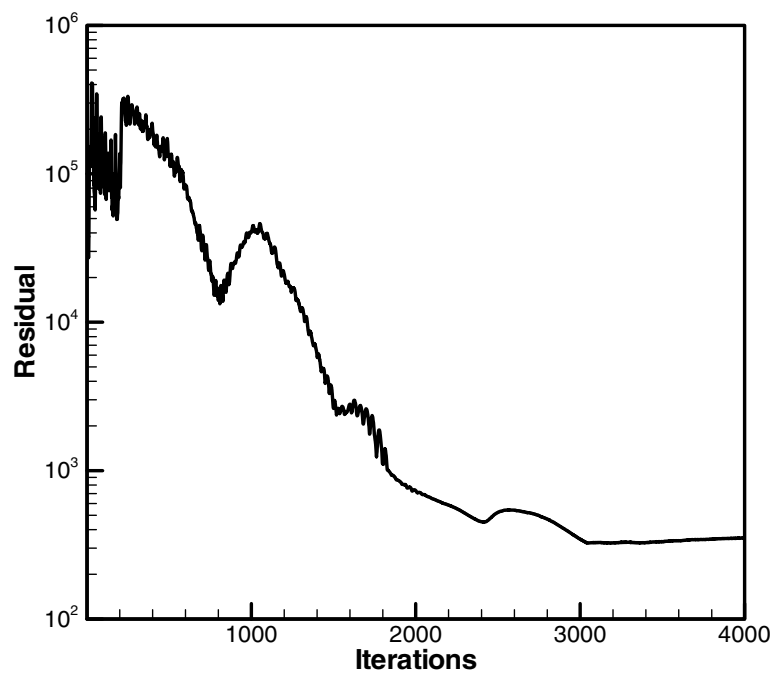

Fig. 2 Convergence history of maximum residual.

history and the computed shock location show the flow to assume a steady state, in contrast to the experiment, in which the flow is observed to show unsteadiness for some NPR values. This difference is not entirely clear. Generally, in a computation with time-stepping methods, convergence without any periodic oscillation in the residual is a good indication that the phenomenon is steady. This is particularly true if one uses a RANS time-averaged computation, which does not handle any random unsteadiness. In the experiment [11], there were no distinct resonant tones observed, which strongly suggests that the unsteadiness is random and hence will not be captured in any unsteady RANS computation. For these reasons, all the computations here are carried out with the steady-state method, in the interest of efficiency.

The typical convergence history of maximum residual of momentum equation at NPR $=1.61$ is presented in Fig. 2. At least two to three orders of residual reductions are obtained. As noted previously, good convergence of a steady computation indicates that no unsteady phenomenon could be calculated with unsteady methods. All the results presented next are obtained by steady-state methods, with Courant-Friedrichs-Lewy numbers varying from 2.0 to 4.0 .

\section{Computational Results and Discussions}

\section{Flow Pattern}

Computed Mach number contours for NPR values from 1.27 to 2.4 are shown in Figs. $3 \mathrm{a}-3 \mathrm{~g}$. Note that the nozzle exit is located at $x / H_{t}=5.2$. It is observed that a well-defined lambda shock appears inside the nozzle for all NPR values. The incident shock, reflected shock, and the triple point characterize each lambda foot for which the incident and reflected shocks merge into the Mach stem. With increasing NPR, the separation point moves downstream of the nozzle throat. One important feature is that for $1.5<\mathrm{NPR}<2.4$, the lambda shock can be asymmetric, with a large foot occurring toward one side of the nozzle. For higher NPR $(\mathrm{NPR}=2.4)$, the shock becomes symmetric. The shock asymmetry is consistent with the experimental observations by Papamoschou and Zill [11], although the computed minimum NPR value (NPR $>1.5$ ) for asymmetric pattern occurring is slightly larger than the experiments at NPR $>1.4$. Typical experimental visualizations of the shock by schlieren photography are compared with the computational counterparts in Figs. 4 and 5.

In the experiments, the asymmetry did not flip sides during a given test run. However, from one experimental run to the next, the asymmetry could change sides. For NPR $>1.5$, only asymmetric separation was observed in the experiment. In the computation, separation is either symmetric or asymmetric, depending on the nature of excitation applied to the initial flowfield. The initial flowfield comprised a uniform inlet velocity with a perturbation of normal injection near the nozzle throat. The side and magnitude of the perturbation can determine the consequent state of the shock separation. It should be noted here that this cross-stream injection was only used in the initial flowfield and not applied throughout the computation. The aim was to initiate an asymmetric mode quickly enough, not to maintain flow asymmetry. Without this initial excitation, the computation takes too long to assume an asymmetric mode via any inherent asymmetry in the numerics. Initial injection velocities of $1,3,5,7.5,10$, and $15 \%$ of the local mean velocity were tried. For injection values greater than $10 \%$, the solution diverged for all NPR values tested. For NPR $\leq 1.5$, only symmetric solutions were found for initial injection velocities of $10 \%$ or below. For $1.5<\mathrm{NPR}<2.4$, symmetric or asymmetric separation was obtained, depending on the magnitude of the initial injection velocity used. Generally, symmetric solutions persisted for initial injection velocities of less than $5 \%$. However, asymmetric solutions were found for injection velocities in the range of 5 to $10 \%$. The asymmetric lambda shock can appear either on the top or bottom wall, depending on the side in which one introduces the injection velocities. It was found that for the same amount of perturbation, the asymmetry becomes rapidly more pronounced with increasing NPR, as shown in Fig. 3 with an injection velocity of $5 \%$. For NPR $\geq 2.4$, only symmetric states were found for all injection velocities tested.

The existence of three shock patterns (one symmetric and two asymmetric) at the same flow and nozzle conditions $\left(A_{e} / A_{t}=1.5\right.$ and NPR $=1.61)$ are shown in Fig. 6. All three different shock patterns are stable, and the axial shock locations are essentially the same. As noted previously, the symmetric state is not observed in the experiments for this NPR. One should keep in mind that the experiment is also affected by an initial field during startup. It is virtually impossible to have perfectly symmetric conditions during the startup process. Thus, it is not surprising, given the symmetric and asymmetric possibilities, that the asymmetric state occurs consistently in the experiment.

It is known from past works that two different separation patterns can exist in overexpanded axisymmetric nozzles: free-shock separation (FSS) or restricted-shock separation (RSS), as observed in the studies of Hagemann et al. [16], Östlund et al. [17], and Östlund and Muhammad-Klingmann [ [18]. In FSS, the nozzle flow fully separates from the wall and the separation region extends downstream from the separation point to the nozzle exit. In contrast, for RSS, the separated flow reattaches to the nozzle wall and the flow becomes supersonic downstream of the reattachment point. Similar separation patterns are obtained here for a planar nozzle, as shown in Fig. 3. For NPR $\leq 1.5$, separation is symmetric and the FSS pattern is obtained for both top and bottom walls. For $1.5<\mathrm{NPR}<2.0$, separation is asymmetric, with the larger separation region accompanied by a larger lambda shock foot. Flow separates at the origin of the incident oblique shock and forms a shear layer that bounds the recirculation region. The reflected branch of the lambda shock further reflects from the shear layer and forms an expansion wave. The asymmetry of the flow could well be the manifestation of the Coanda effect, whereby a jet surrounded by, or adjacent to, a surface attaches to that surface. At NPR $=2.4$, the separation line approaches the nozzle exit and the recirculation zone opens to the ambient region. The separation pattern transits from RSS back to FSS, causing the overall flow pattern to become symmetric. This phenomenon, sometimes referred to as an "end effect," is commonly found in supersonic nozzle flow [18]. To provide more details of the asymmetric separation, Fig. 7 plots the streamline pattern through the shock and iso-Mach number contours in the large separation region. As in Fig. 3e, the FSS and RSS regions are evident.

The evolution of the shock area ratio $A_{s} / A_{t}$ versus the pressure ratio is presented in Fig. 8 , in which the present computational results are compared with both experimental data and one-dimensional inviscid theory. Here, $A_{s}$ is defined as the area corresponding to the axial position of the Mach stem (normal shock). As seen from the figure, the computations are in good agreement with the experiments. The scatter of the experimental data around NPR $=1.60$ is caused by the unsteadiness of the shock motion that our computation does not capture, as discussed earlier. The experimental data relied on 


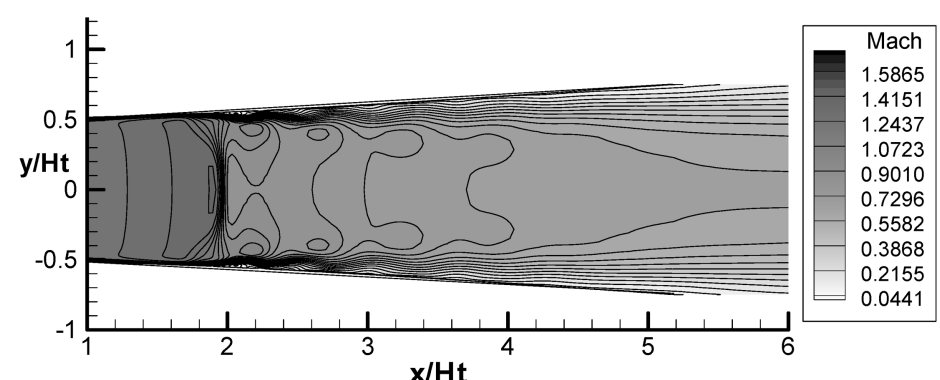

a)

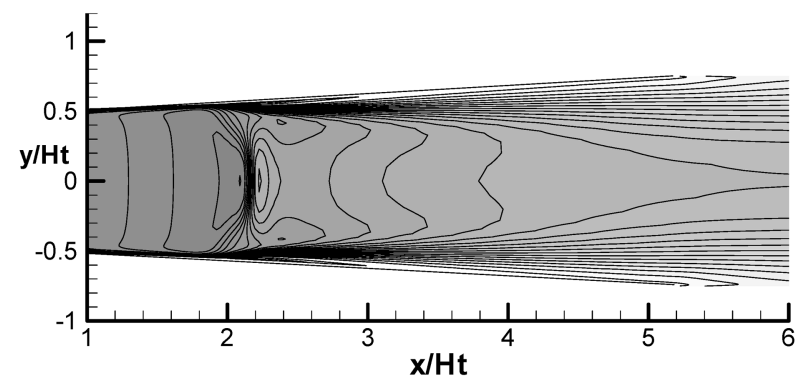

b)

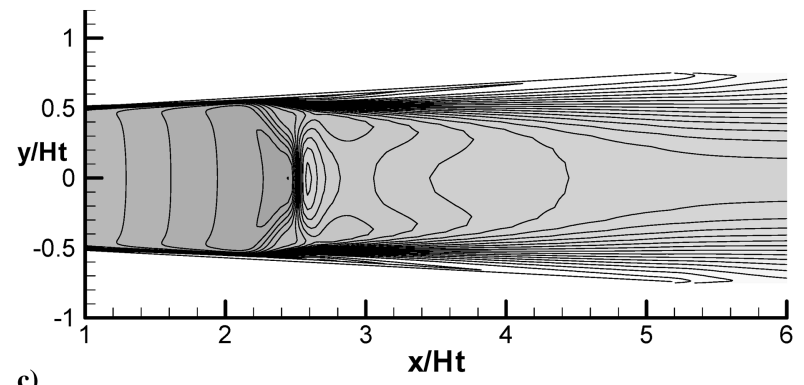

c)

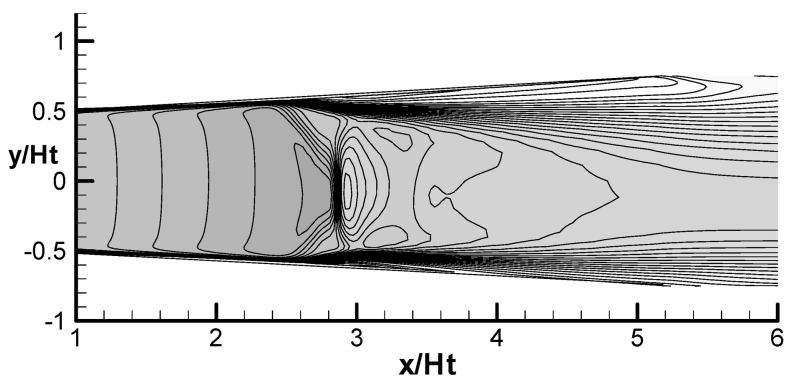

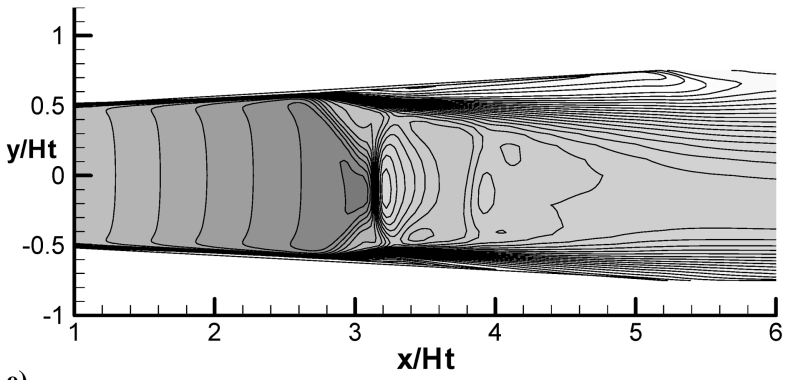

e)

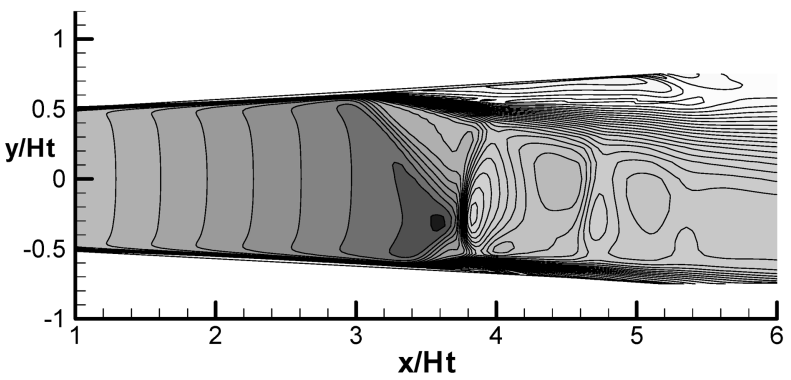

f)

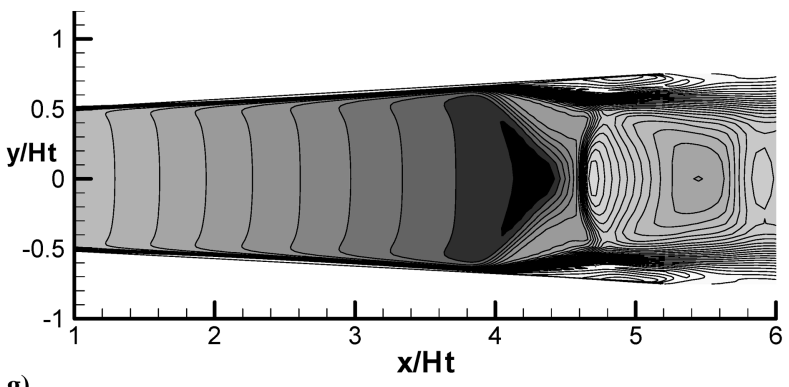

g)

d)

Fig. 3 Mach number contours at different NPR values: a) $N P R=1.27$, b) $N P R=1.34$, c) $N P R=1.47, d) ~ N P R=1.61, e) ~ N P R=1.70$, f) $N P R=2.0$, and g) $\mathrm{NPR}=2.4$.

instantaneous flow visualization pictures, thus capturing the unsteady motion of the shock. The discrepancy of the experimental and computational results with the one-dimensional theory is substantial. The shock sits at a station in which the area ratio is much smaller than that predicted by one-dimensional theory. The theory predicts that flow past the shock recovers (compresses) monotonically to the ambient pressure. In reality, the flow past the Mach stem first expands and then compresses, as we shall see from our computations in the later part of this paper. The shock feels a local back pressure higher than the theoretical value and thus sits upstream of the theoretically predicted location.

\section{Static Pressure Distribution}

In presenting the wall pressure distributions, given the flow asymmetry, we need to distinguish between the wall with the larger separation zone and the wall with the smaller separation zone. We begin here by showing the pressure distribution on the wall with the smaller separation zone, plotted in Fig. 9. The computed pressures agree well with the experimental measurements. The pressure distributions have the classic shape of expansion, shock jump, and recovery. For lower NPRs, the pressure downstream of the shock recovers to the ambient pressure in a gradual way. For NPR $=2.0$ and 2.4 , the pressure recovery is more immediate.

Next, we compare the pressure distributions on the top and bottom walls, shown in Fig. 10. For low NPR values such as 1.27 , because the lambda shock is symmetric, there are no noticeable differences between the top and bottom walls. However, for a large NPR such as 1.61 , subtle differences exist between the two walls as a result of the asymmetric shock pattern. On the side of the larger separation zone (top wall), the pressure recovers linearly with axial distance. On the side of the small separation zone (bottom wall), the pressure shows a faster initial rise, followed by a gradual recovery to ambient value on the bottom wall on which the smaller foot occurs. Papamoschou and Zill [11] found the same behavior in their experiments. The difference in pressure distribution on the top and bottom walls is consistent with the asymmetric separation pattern discussed in the previous section. The typical characteristic of wall pressure distribution for FSS and RSS is more clearly reflected in Fig. 11 at 


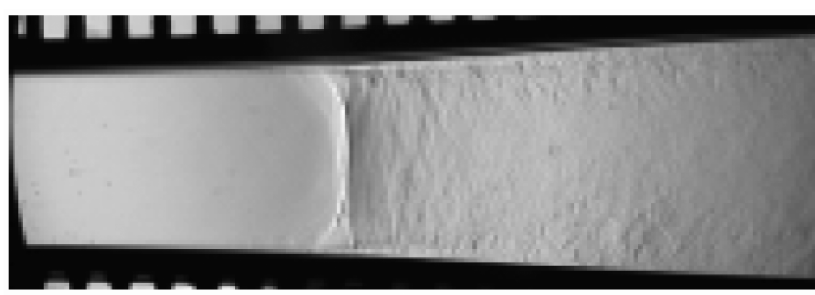

a)

Fig. 4 Comparison of the flow pattern for a symmetric lambda shock at NPR = 1.33: a) experimental spark schlieren flow visualization [11] and b) computed schlieren picture.

$\mathrm{NPR}=2.0$.The separation point for the smaller separation zone (bottom, RSS) locates further downstream than in the large separation zone (top, FSS). The pressure rise across the shock is larger for the smaller separation zone than for the larger separation zone.

The pressure distribution along the centerline of the nozzle, shown in Fig. 12, is very different from the wall pressure distribution, but is consistent with the measurements of Papamoschou and Zill [11]. Downstream of the Mach stem, the flow expands rapidly and then compresses. Romine's [6] hypothesis of flow recovery via an underexpansion is thus only partly valid. Our computational results show that the underexpansion is a localized phenomenon and is always followed by a compression zone. Detailed examination of the flowfield in the next section will show that the flow compression is a

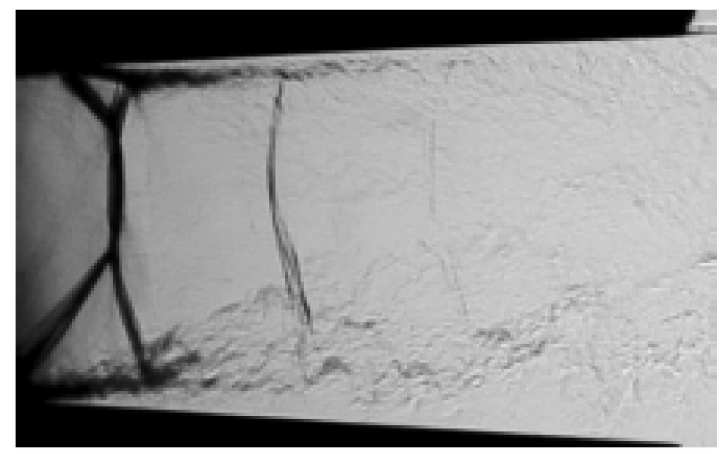

a)

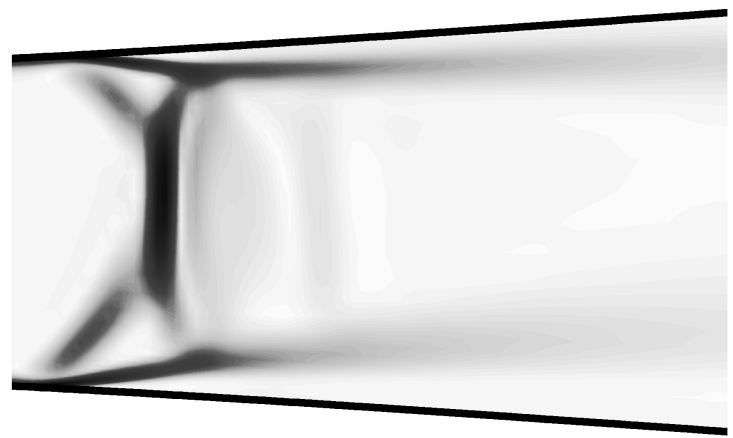

b)

Fig. 5 Comparison of the flow pattern for an asymmetric lambda shock with a large foot at the bottom wall: a) experimental spark schlieren visualization (NPR = 1.50 [11]) and b) computed schlieren picture $(\mathrm{NPR}=1.54)$.

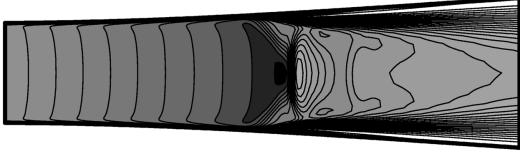

1.4500

1.2500

1.0500

0.8500

0.6500

0.4500

0.2500

0.0500

a)

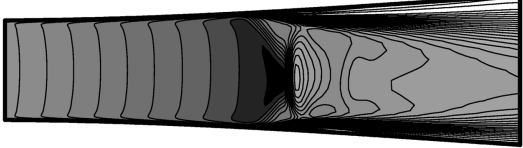

b)

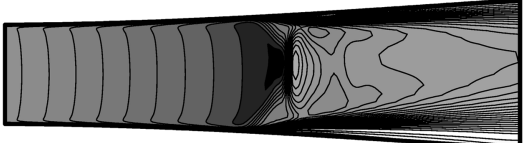

c)

Fig. 6 Mach number contours for three different shock waves at $A_{e} / A_{t}=1.5$ and $\mathrm{NPR}=1.61$ : a) symmetric shock, b) asymmetric lambda shock with a large foot at the top wall, and c) asymmetric lambda shock with a large foot at the bottom wall.

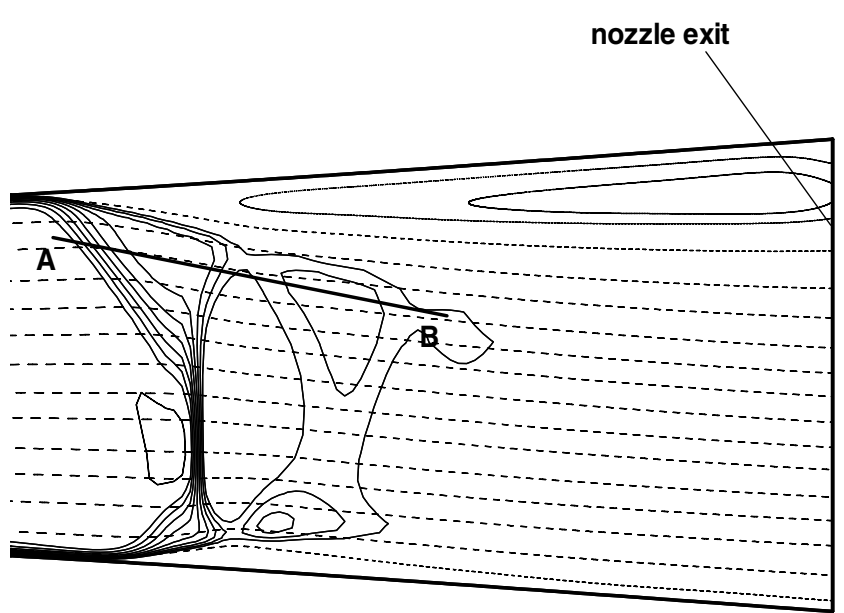

Fig. 7 Streamline pattern and Mach number contours for separated flow with NPR $=1.70$ [dashed lines are streamlines; solid lines are Mach number contours (minimum is $\mathbf{1 . 0 0}$, maximum is 1.52 , and the interval is 0.2)].

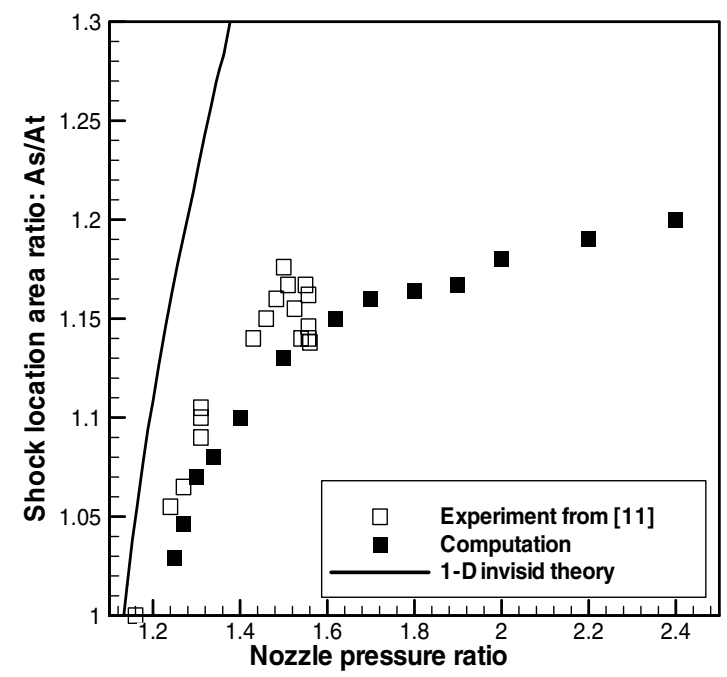

Fig. 8 Shock location versus nozzle pressure ratio. 


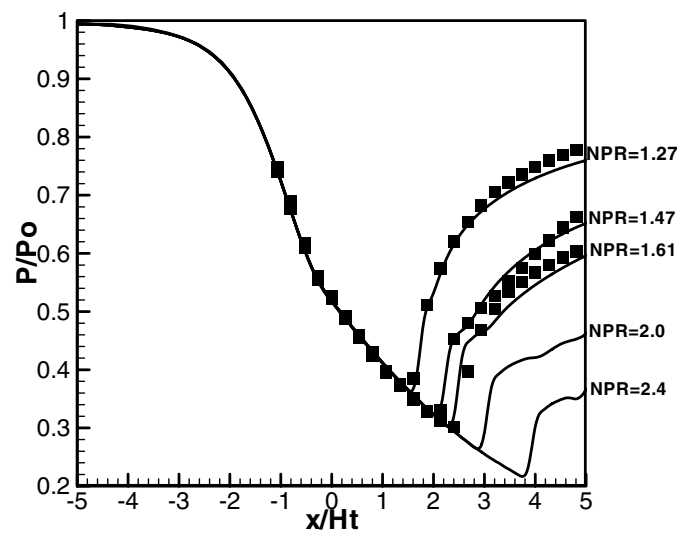

Fig. 9 Pressure distribution on the wall with the small separation zone; symbols indicate experiments [11] and lines indicate computation.

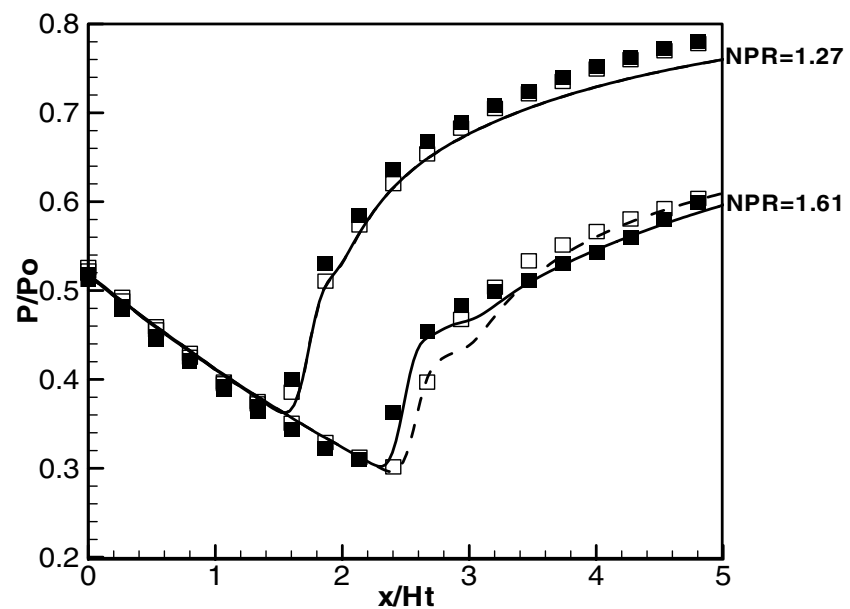

Fig. 10 Top and bottom wall pressure distribution at NPR $=1.27$ and 1.61; top wall has large separation zone for $N P R=1.61$; lines indicate computation (dash, bottom wall; solid, top wall) and symbols indicate experiments [11] (open symbols, top wall; solid symbols, bottom wall).

consequence of the expansion wave. Our computation and Romine's model are consistent in that an underexpansion creates a large back pressure for the shock, which causes the shock to sit closer to the throat than one would predict using one-dimensional theory.

\section{Shock Structure}

In this section, we discuss in detail the wave and fluid phenomena associated with flow separation. The computational results of Fig. 3

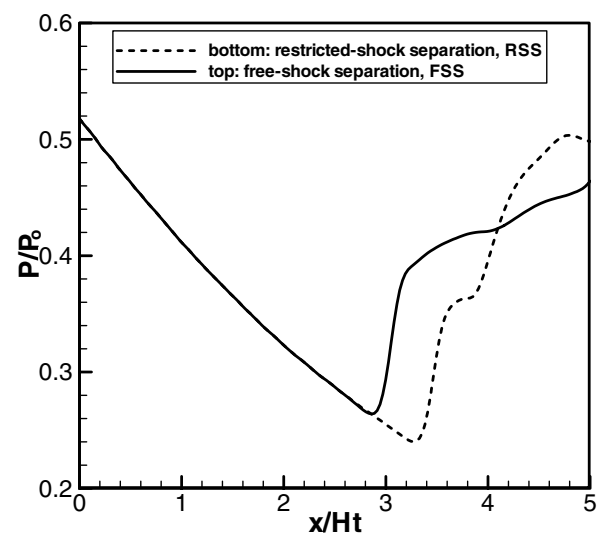

Fig. 11 Top and bottom wall pressure distribution for NPR $=2.0$ with free-shock separation on the top wall and restricted-shock separation on the bottom wall.

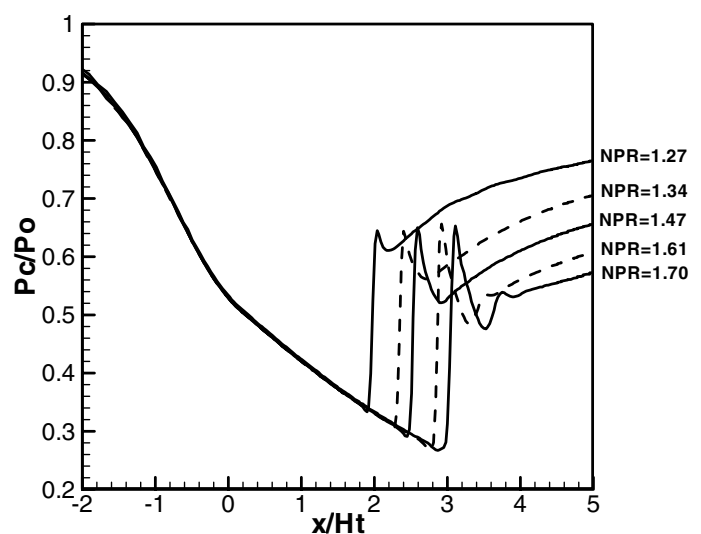

Fig. 12 Static pressure distribution along the nozzle centerline.

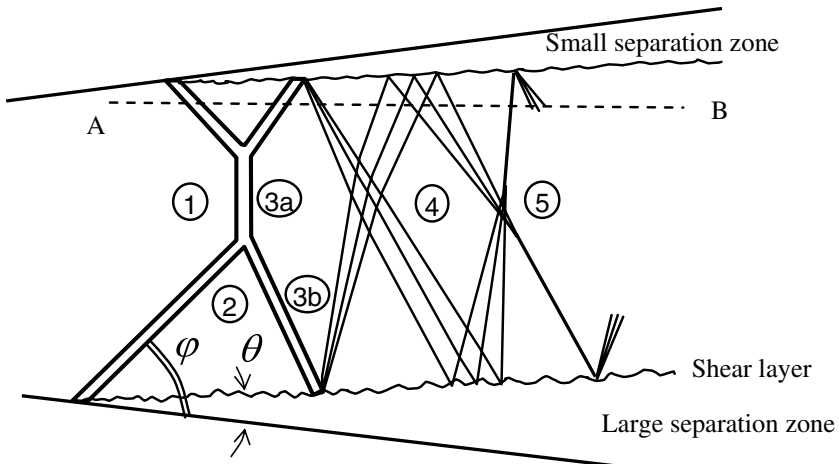

Fig. 13 Simplified schematic of the shock and flow structure.

and the experimental pictures of Fig. 5 reveal a succession of weak waves past the main shock for large NPRs. The flow immediately downstream of the main shock accelerates to supersonic speed, recompresses, and then reaccelerates. The sketch of the successive shock pattern and flow structure as proposed by Papamoschou and Zill [11] is recreated and amplified in Fig. 13. Subscripts 1 and 2 denote the conditions before the Mach stem and after the incident oblique shock, respectively. Subscripts $3 \mathrm{a}$ and $3 \mathrm{~b}$ represent the conditions after the Mach stem and reflected oblique shock, respectively. Subscripts 4 and 5 represent the condition after the expansion and compression, respectively, following the main shock. To quantify the interaction between the waves and the separation shear layer, Table 1 lists the Mach numbers in the aforementioned regions and the basic parameters of the shock geometry. $H_{s}$ is the height of the Mach stem and $H_{t}$ is the height of the throat. Carroll and Dutton $[19,20]$ previously observed similar phenomena that they termed as "multiple normal shock train" in a nearly constant area rectangular with undisturbed Mach number of 1.61. They found a symmetric shock structure, in contrast with the experiments of Papamoschou and Zill [11] and with results of the present computation that reveal the likelihood of asymmetric separation.

Mach number distributions along the center plane of the main Mach stem for different NPRs are shown in Fig. 14. The expansion (acceleration) immediately after the Mach stem is clearly seen for all NPRs. Because of the rapidity of this expansion, the Mach number $M_{3 a}$ immediately past the shock is not resolved accurately in the computation and thus is slightly higher than that given by the normal shock relations. Successive recompression and expansion waves are obtained for larger NPRs such as 1.61,1.70, and 2.0. Figure 15 shows the Mach number distribution along the center plane of the Mach stem and along the $A-B$ plane (shown in Figs. $\underline{7}$ and 13 ) for $\mathrm{NPR}=1.70$.

The effect of NPR on the shock structure can be seen in Table 1 and Figs. 14 and 15. The Mach numbers immediately before the $\bar{M}$ ach stem $\left.\overline{(M}_{1}\right)$, after the incident shock $\left(M_{2}\right)$, after the reflected shock 
Table 1 Effect of nozzle pressure ratio on the shock structure

\begin{tabular}{|c|c|c|c|c|c|c|c|c|c|c|c|c|c|c|}
\hline \multirow[b]{2}{*}{ NPR } & \multirow[b]{2}{*}{$M_{1}$} & \multirow[b]{2}{*}{$M_{3 a}$} & \multirow[b]{2}{*}{$M_{4}$} & \multirow[b]{2}{*}{$M_{5}$} & \multirow[b]{2}{*}{$H_{s} / H_{t}$} & \multicolumn{4}{|c|}{ Large separation zone } & \multicolumn{4}{|c|}{ Small separation zone } & \multirow[b]{2}{*}{$\overline{\text { Separation }}$} \\
\hline & & & & & & $\varphi$ & $\theta$ & $M_{2}$ & $M_{3 b}$ & $\varphi$ & $\theta$ & $M_{2}$ & $M_{3 b}$ & \\
\hline 1.27 & 1.23 & 0.810 & - & - & 0.637 & $40 \mathrm{deg}$ & $6 \mathrm{deg}$ & 1.08 & 0.86 & $40 \mathrm{deg}$ & $6 \mathrm{deg}$ & 1.08 & 0.86 & Symmetric \\
\hline 1.34 & 1.39 & 0.795 & 0.93 & - & 0.545 & $48 \mathrm{deg}$ & $8 \mathrm{deg}$ & 1.12 & 0.90 & $48 \mathrm{deg}$ & $8 \mathrm{deg}$ & 1.12 & 0.90 & Symmetric \\
\hline 1.47 & 1.44 & 0.780 & 0.95 & - & 0.509 & $48 \mathrm{deg}$ & $11 \mathrm{deg}$ & 1.16 & 0.93 & $48 \mathrm{deg}$ & $11 \mathrm{deg}$ & 1.16 & 0.93 & Symmetric \\
\hline 1.61 & 1.50 & 0.770 & 1.05 & 0.96 & 0.454 & $50 \mathrm{deg}$ & $13 \mathrm{deg}$ & 1.16 & 1.02 & $45 \mathrm{deg}$ & $10 \mathrm{deg}$ & 1.22 & 1.04 & Asymmetric \\
\hline 1.70 & 1.56 & 0.750 & 1.10 & 0.94 & 0.424 & $52 \mathrm{deg}$ & $15 \mathrm{deg}$ & 1.21 & 1.02 & $47 \mathrm{deg}$ & $13 \mathrm{deg}$ & 1.31 & 1.04 & Asymmetric \\
\hline 2.0 & 1.65 & 0.740 & 1.19 & 0.93 & 0.409 & $48 \mathrm{deg}$ & $11 \mathrm{deg}$ & 1.28 & 1.03 & $45 \mathrm{deg}$ & $10 \mathrm{deg}$ & 1.36 & 1.06 & Asymmetric \\
\hline 2.3 & 1.67 & 0.825 & - & - & 0.31 & $43 \mathrm{deg}$ & $11 \mathrm{deg}$ & 1.30 & 1.03 & $40 \mathrm{deg}$ & $10 \mathrm{deg}$ & 1.36 & 1.07 & Asymmetric \\
\hline 2.4 & 1.69 & 0.840 & - & - & 0.10 & 42 deg & $8 \mathrm{deg}$ & 1.40 & 1.05 & $42 \mathrm{deg}$ & $8 \mathrm{deg}$ & 1.40 & 1.07 & Symmetric \\
\hline
\end{tabular}

$\left(M_{3 b}\right)$, and after the expansion wave $\left(M_{4}\right)$ all increase with increasing NPR. For all NPRs, the flow after the incident oblique shock $\left(M_{2}\right)$ is supersonic. In the range $1.5<\mathrm{NPR}<2.4$, the lambda shock is asymmetric, therefore $M_{2}$ on the large lambda foot side is always smaller than $M_{2}$ on the small lambda foot, because of the larger flow deflection $\theta$ on the side of the larger separation zone. For NPR $>1.47$, the reflected shock is of the weak type, thus the Mach number after the reflected shock $\left(M_{3 b}\right)$ is supersonic. The resulting entropy layer between regions $3 \mathrm{a}$ and $3 \mathrm{~b}$ becomes stronger as NPR increases. Given that $M_{3 b}$ is supersonic, the reflected shock reflects from the shear layer as an expansion wave, which accelerates the flow past the Mach stem to supersonic speed $\left(M_{4}>M_{3 b}>1\right)$. The expansion wave reflects from the shear layer as a compression wave (eventually coalescing into a shock) and decelerates the flow to a high subsonic Mach number $\left(M_{5}<1\right)$. This trend is more obvious for larger NPRs, as seen in Fig. 14. Generally, two to three successive expansion/compression systems past the main shock are observed from the computation. For NPR $=2.3$ and 2.4, as the shock sits near the exit of nozzle, the compression and expansion waves occur

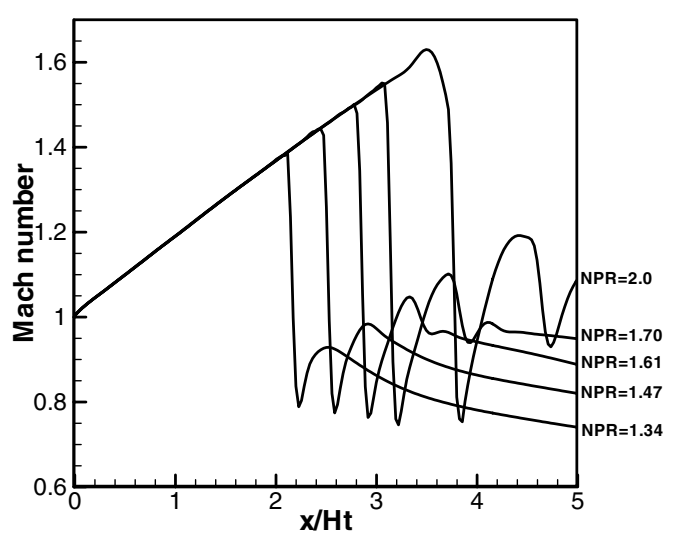

Fig. 14 Mach number distribution along the center plane of the Mach stem.

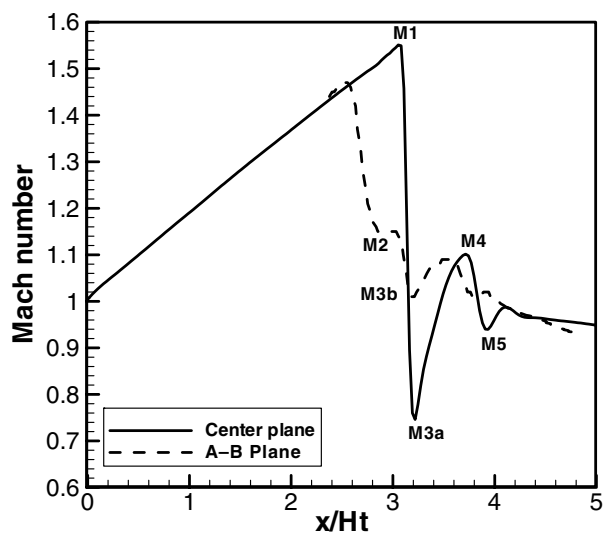

Fig. 15 Mach number distributions along the center plane of the main Mach stem and the $A-B$ plane (shown in Figs. 7 and 13) for $N P R=1.70$. downstream of the nozzle exit. The shock angle $\varphi$ and the deflection angle $\theta$ increase with NPR and then decrease. The height of the Mach stem decreases monotonically with increasing NPR, which is similar to the experimental observations.

Finally, we discuss the behavior of the turbulent kinetic energy, $k=\frac{1}{2}\left(\overline{u^{\prime 2}}+\overline{v^{\prime 2}}+\overline{w^{\prime 2}}\right)$, normalized by the square of the perfectly expanded exit velocity $U_{\mathrm{pe}}$. The normalization removes the obvious effect of increasing $k$ with increasing velocity of the separated flow. Figure 16 shows the variation of the $k / U_{\mathrm{pe}}^{2}$ with NPR. The normalized turbulent kinetic energy peaks inside the separation shear layers and is greater in the shear layer surrounding the larger separation zone. Toward the exit of the nozzle, the level of $k / U_{\mathrm{pe}}^{2}$ is observed to drop. In the central region of the nozzle, the fluctuation levels are very small. With increasing NPR from 1.27 to 2.4 , the maximum level of the normalized turbulent intensity level increases slightly (by about 7\%) and the location of the maximum turbulent intensity moves toward the nozzle exit plane.

\section{Conclusions}

The separated flow in a convergent-divergent nozzle is studied computationally for a nozzle of an exit-to-throat area ratio $A_{e} / A_{t}$ of 1.5 and over a range of nozzle pressure ratios. The computations use the Reynolds-averaged Navier-Stokes equations with a twoequation $k-\omega$ turbulence model to examine the flow physics of asymmetric separated flow in a symmetric nozzle. The experimental results from Papamoschou and Zill [11] are used as benchmark to assess the computational results. The following conclusions can be drawn:

1) Unlike in the experiment, no unsteady shock movements were captured, despite initial attempts to time-resolve any unsteady motion in the flow. In all computations made here, the flow converges to a steady state. This may be in line with the experimental observation that there is no distinct resonant tone, indicating a possible lack of acoustic feedback mechanism. The inability of the RANS computation to capture the unsteady motion of the shock may well confirm that the motion is indeed random.

2) For $1.5<\mathrm{NPR}<2.4$, the computation reveals the possibility of symmetric and asymmetric separation, depending on the initial flowfield. The asymmetric flow separation structures computed are consistent with experimental observations. For higher values of NPR, for which the shock sits closer to the nozzle exit, only symmetric separation is formed.

3) The computed wall pressure distribution is in good agreement with experimental data, which show a gradual pressure recovery to ambient pressure after the separation shock. Subtle changes in the recovery due to asymmetric separations were captured accurately by the computations. The static pressure distribution along the nozzle centerline shows a rapid flow expansion past the Mach stem, followed by compression.

4) Consistent with the centerline static pressure distribution, flow immediately downstream of the separation Mach stem expands rapidly to a higher velocity, contrary to the prediction of onedimensional theory. For NPR $>1.5$, this higher velocity is supersonic and the flow is subjected to a succession of expansion and compression waves. Expansion past the Mach stem causes the shock to sit at an area ratio much smaller than that predicted by 


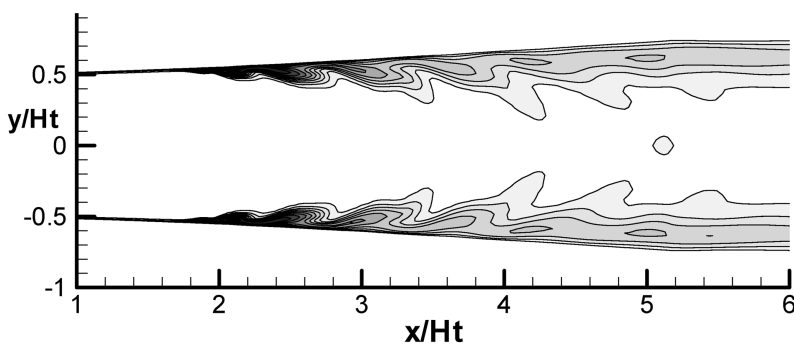

a)

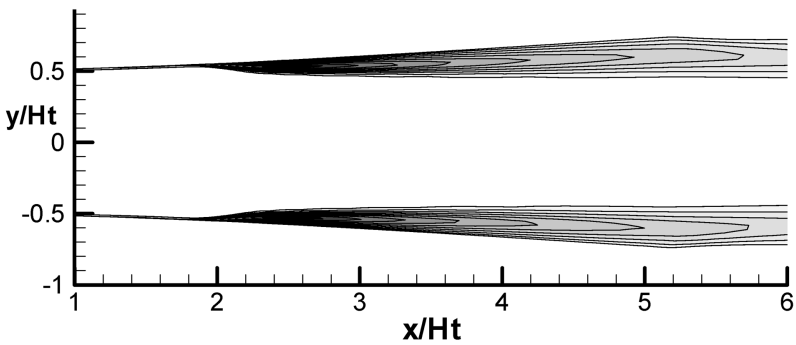

b)

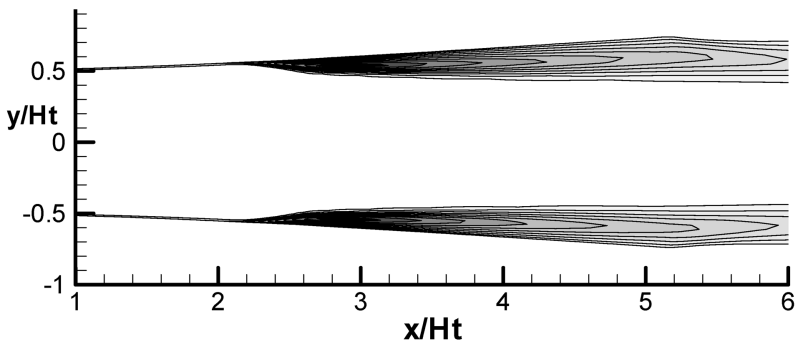

c)

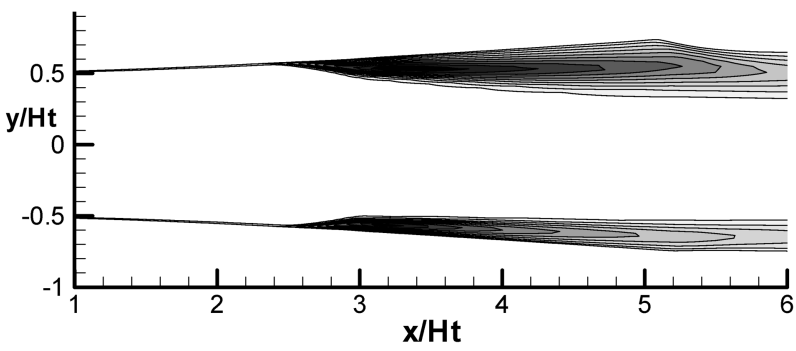

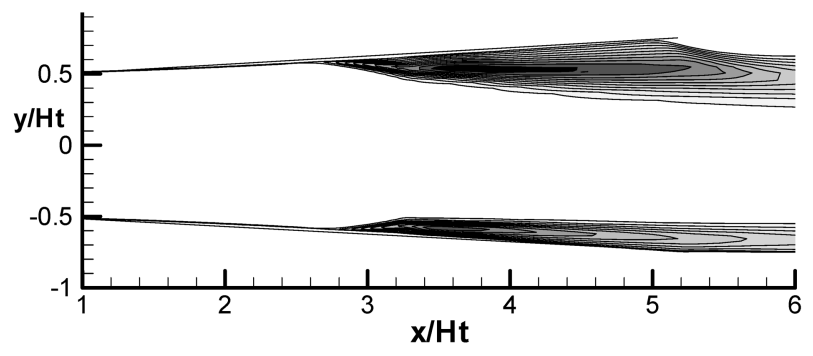

e)

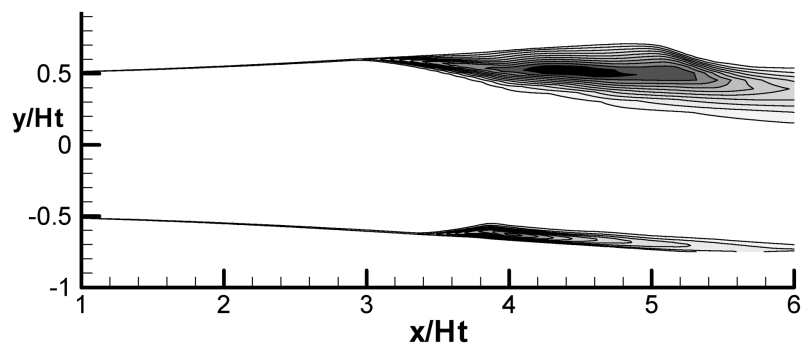

f)

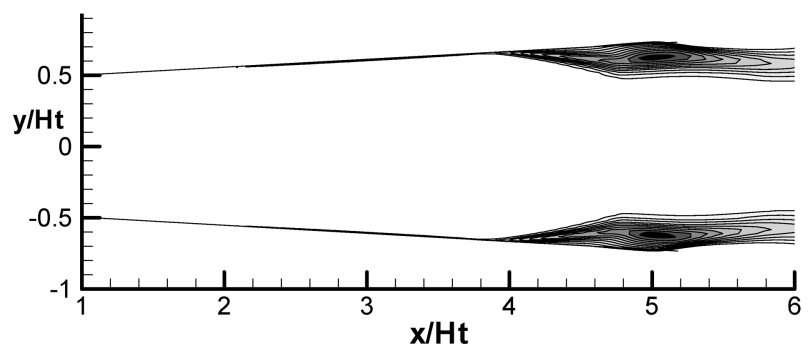

g)

d)

Fig. 16 Normalized turbulent kinetic energy $k / U_{\mathrm{pe}}^{2}$ contours at different NPRs: a) $\mathrm{NPR}=1.27$, b) $\mathrm{NPR}=1.34$, c) $\mathrm{NPR}=1.47$, d) $\mathrm{NPR}=1.61$, e) $\mathrm{NPR}=1.70, \mathrm{f}) \mathrm{NPR}=\mathbf{2 . 0}$, and $\mathrm{g}$ ) $\mathrm{NPR}=2.4$ (minimum is 0.0074 , maximum is 0.74 , and the interval is 0.022 ).

one-dimensional theory. More important, the interaction of the successive waves with the separation shear layer could be a critical ingredient for the violent instability of the separation shear layer observed in the experiments.

5) For the asymmetric case, the larger separation zone dominates the nozzle flow past the shock and exhibits higher turbulence levels than the smaller separation zone. From the experiments, the shear layer enclosing the larger separation zone is known to be highly unstable and caused very large eddies to be formed inside and outside the nozzle. The RANS computation presented here captures this phenomenon, albeit in a statistical sense. As our interest is to exploit this phenomenon as a means for fluidic actuation to enhance jet mixing, the capability of the method to compute this flow is important. This allows us to use this as a tool to assess such fluidic phenomenon and can be further used to predict the unsteadiness of the plume exiting the nozzle.

\section{References}

[1] Papamoschou, D., "Mixing Enhancement Using Axial Flow," AIAA Paper 2000-0093, Jan. 2000.

[2] Murakami, E., and Papamoschou, D., "Experiments on Mixing Enhancement in Dual-Stream Jets," AIAA Paper 2001-0668, Jan. 2001.
[3] Papamoschou, D., Dixon, T. D., and Nishi, K., "Mean Flow of MultiStream Rectangular Jets Under Normal and Mixing-Enhancement Conditions," AIAA Paper 2004-0919, Jan. 2004.

[4] Morrisette, E. L., and Goldberg, T. J., "Turbulent Flow Separation Criteria for Over-Expanded Supersonic Nozzles," NASA TP 1207, Aug. 1978.

[5] Reshotko, E., and Tucker, M., "Effect of a Discontinuity on Turbulent Boundary Layer Thickness Parameters with Application to ShockInduced Separation," NACA TN-3454, 1955.

[6] Romine, G. L., "Nozzle Flow Separation," AIAA Journal, Vol. 36, No. 9, 1998, pp. 1618-1625.

[7] Hunter, C. A., "Experimental, Theoretical, and Computational Investigation of Separated Nozzle Flows," AIAA Paper 98-3107, 1998.

[8] Carlson, J. R., "A Nozzle Internal Performance Prediction Method," NASA TP 3221, 1992.

[9] Xiao, Q., Tsai, H. M., and Liu, F., "Computation of Transonic Diffuser Flows by a Lagged k- $\omega$ Turbulence Model," Journal of Propulsion and Power, Vol. 19, No. 3, 2003, pp. 473-483.

[10] Xiao, Q., Tsai, H. M., and Liu, F., "Computation of Turbulent Separated Nozzle Flow by a Lag Model," Journal of Propulsion and Power, Vol. 21, No. 2, 2005, pp. 368-371.

[11] Papamoschou, D., and Zill, A., "Fundamental Investigation of Supersonic Nozzle Flow Separation,” AIAA Paper 2004-1111, Jan. 2004 
[12] Bourgoing, A., and Reijasse, P., "Experimental Analysis of Unsteady Flows in a Supersonic Planar Nozzle," International Symposium on Shock Waves, Vol. 14, No. 4, Springer, New York, 2001, pp. 251-258.

[13] Reijasse, P., and Bourgoing, A., "Unsteady and Asymmetry of ShockInduced Separation in a Planar Two-Dimensional Nozzle," AIAA Paper 99-3694, 1999.

[14] Deck, S., Hollard, R., and Guillen, Ph., "Numerical Simulation of Steady and Unsteady Separated Nozzle Flows," AIAA 2002-0406, 2002.

[15] Sadeghi, M., Yang, S., Liu, F., and Tsai, H. M., "Parallel Computation of Wing Flutter with a Coupled Navier-Stokes and CSD Method," AIAA Paper 2003-1347, 2003.

[16] Hagemann, G., Frey, M., and Koschel, W., "Appearance of Restricted Shock Separation in Rocket Nozzles," Journal of Propulsion and Power, Vol. 18, No. 3, 2002, pp. 577-584.
[17] Östlund, J., Damgaard, T., and Frey, M., "Side-Load Phenomena in Highly Overexpanded Rocket Nozzle," Journal of Propulsion and Power, Vol. 20, No. 4, 2004, pp. 695-704.

[18] Östlund, J., and Muhammad-Klingmann, B., "Supersonic Flow Separation with Application to Rocket Engine Nozzle," Applied Mechanics Reviews, Vol. 58, May 2005, pp. 143-176.

[19] Carroll, B. F., and Dutton, J. C., "Multiple Normal Shock Wave/ Turbulent Boundary-Layer Interactions," Journal of Propulsion and Power, Vol. 8, No. 2, 1992, pp. 441-448.

[20] Carroll, B. F., and Dutton, J. C., "Turbulence Phenomena in a Multiple Normal Shock Wave/Turbulent Boundary-Layer Interaction," AIAA Journal, Vol. 30, No. 1, 1992, pp. 43-48.

C. Kaplan

Associate Editor 